\title{
Correction to: Non-alcoholic Wernicke's encephalopathy with cortical involvement and polyneuropathy following gastrectomy
}

\author{
Wei-Chia Tsao ${ }^{1}$. Long-Sun $\mathrm{Ro}^{2}$ • Chiung-Mei Chen ${ }^{2} \cdot$ Hong-Shiu Chang $^{2}$. \\ Hung-Chou Kuo ${ }^{2}$
}

Published online: 19 October 2017

(C) Springer Science+Business Media, LLC 2017

Correction to: Metab Brain Dis (2017) 32:1649-1657

https://dx.doi/10.1007/s11011-017-0055-8

In the original publication of the article, author name Hong-

Shiu Chang was incorrectly written as Hong-Chiu Chang.

The authors regret the oversight.

The online version of the original article can be found at https://dx.doi/10. 1007/s11011-017-0055-8

\footnotetext{
Hung-Chou Kuo

kuo0426@adm.cgmh.org.tw

1 Department of Neurology, Kaohsiung Medical University Hospital, Kaohsiung Medical University, Kaohsiung, Taiwan

2 Section of Neuromuscular Disorders, Department of Neurology, Chang Gung Memorial Hospital at Linkou Medical Center and Chang Gung University College of Medicine, 5 Fuhsing Street, Kueishan, Taoyuan, Taiwan
} 